\title{
Targeted Jumping of Compliantly Actuated Hoppers based on Discrete Planning and Switching Control
}

\author{
Dominic Lakatos, Daniel Seidel, Werner Friedl, and Alin Albu-Schäffer
}

\begin{abstract}
We address the operation of robotic legs with intrinsic elasticity in hopping cycles determined by the mechanical resonant properties of the system. This ensures energy efficiency and high jumping velocity and distance. Recently, we have shown in simulation that a simple, biologically inspired bang-bang controller operating in the local coordinate of the first resonant mode leads to limit cycles which are robust with respect to leg model uncertainties and ground properties. In this paper we address the velocity control of the hopping and the planning of the bang-bang control parameters for the case that the systems should not move at steady state velocity, but should have different step lengths and heights. We exploit the discrete structure and the small number of parameters of the controller to develop a fast optimization procedure for generating an arbitrary sequence of steps. This approach can provide high motion performance, robustness and substantial computational time saving compared to continuous trajectory and controller gain planning. The stationary and the aperiodic hopping is validated by experiments on a new planar elastic leg.
\end{abstract}

\section{INTRODUCTION}

Taking advantage of compliant actuation to increase the velocity of locomotion robots is a topic of very active research [1], [2], [3], [4], [5], [6], [7], [8]. The approach is motivated by the elastic properties of muscles and tendons which are known to be responsible in biological systems for energy efficiency and robustness at the impact with uneven ground. Solutions of increasing complexity have been for example developed starting from the simple pogo-stick model and extending it to include more detailed effects such as leg segmentation and trunk dynamics [9], [10]. Still, the biological mechanisms to optimally take advantage of the elasticity are not yet completely understood. Designing controllers, which impose these idealized models to real robots is also still a challenge in view of uncertain models and actuator limitations. From engineering point of view, exciting and sustaining nonlinear limit cycles in the highly coupled, nonlinear dynamics of elastic bodies while exploiting to the maximal extend possible the natural resonance properties are theoretically still not completely solved problems. Same is valid for non-periodic motions, for which computationally intensive nonlinear optimization needs to be used to solve the optimal control problems for the multi-dof case [11], [12], [13], [14].

In our previous work we designed a bang-bang control approach, which requires kinematic and only rough dynamic

The authors are with the Institute of Robotics and Mechatronics, German Aerospace Center (DLR), D-82234 Oberpfaffenhofen, Germany. Alin Albu-Schäffer and Daniel Seidel is also with Technical University Munich, Chair of Sensor Based Robots and Intelligent Assistance Systems, Department of Informatics, D-85748 Garching, Germany. Contact: dominic.lakatosedlr.de models of the robot and proved to be very effective to stabilize limit cycles in various robotic systems by simulation and experiments [15], [16]. The main idea is to excite the local principal resonance mode of the whole body dynamics by introducing a constant amount of energy in a cotangent space direction (generalized torque) corresponding to the modal coordinate. We have shown that this is even a biologically plausible approach, which might in principle be implemented by only a few neurons [17].

In this paper we address the questions of regulating the velocity of the stationary limit cycle on even ground and of planning the control parameters for aperiodic hopping motions for uneven terrain. Due to the discrete nature of the controller and the small number of parameters, the optimization of hopping reduces to a computationally efficient discrete time problem. For validating the concepts we developed a rapid prototyped biarticular leg with elastic transmission. We briefly introduce the new leg prototype and verify the periodic and aperiodic motion control in experiments. Even though, the presented method is introduced for a single leg, it can be straightforwardly extended to the case of multiple legs by considering the grouping concept of virtual legs as introduced in [1].

\section{THE IDEA}

This methodology is the result of recent developments in the mechanical design and control of compliantly actuated robots. It merges the following advantageous properties: (i) mechanical robustness of compliant actuators, (ii) energy efficiency due to directed buffering and releasing elastic energy, (iii) parameter robustness of switching based limit cycle control, and (iv) the reduced complexity of discrete planning such that the approach can be applied to rather imprecisely machined, rapid prototyping robotic systems suffering from noisy sensing. This way, we satisfy the initially controversially sounding requirements low-cost and efficiency.

\section{A. Compliant actuators}

The basic idea of the approach will be first explained for a single compliantly actuated joint. Consider therefore the motor inertia $B>0$ acting on the load inertia $M>0$ via a spring

$$
\tau(\theta, q)=\frac{\partial U(\theta, q)}{\partial q},
$$

where $\theta \in \mathbb{R}$ and $q \in \mathbb{R}$ represent motor and joint coordinate, respectively and the potential $U$ is an even and positive 
definite function of the spring deflection $\phi=\theta-q$ such that the corresponding dynamics takes the form

$$
\begin{aligned}
B \ddot{\theta}+\tau(\theta, q) & =u+\tau_{\mathrm{BF}} \\
M \ddot{q} & =\tau(\theta, q)+\tau_{\mathrm{MF}}+\tau_{\mathrm{ext}} .
\end{aligned}
$$

1) Mechanical robustness: For simplicity, consider a linear spring of the form $\tau=K(\theta-q)$ with stiffness $K>0$ and let the friction acting on the load be $\tau_{\mathrm{MF}}=-D \dot{q}$ with damping $D>0$ such that the dynamics of the load results in

$$
M \ddot{q}+D \dot{q}+K q=K \theta+\tau_{\mathrm{ext}} .
$$

By fixing the motor position $\theta$ it can be seen that any abrupt changes in the external force $\tau_{\text {ext }}$ (impacts) evolve smoothly (second order low-pass filtered) in the spring force $\tau$ acting on the motor. This demonstrates (i) the mechanical robustness of compliant actuators, in which the gear box and the motor are protected from impacts.

2) Separating the link side dynamics from the motor: In the following, we assume that the motor position $\theta$ is a control input. This assumption can be achieved by considering the PD control $u=-K_{\mathrm{P}}\left[\theta-\theta_{\mathrm{d}}\right]-K_{\mathrm{D}} \dot{\theta}-\tau_{\mathrm{BF}}$ in (2), where $K_{\mathrm{P}}, K_{\mathrm{D}}>0$ are controller gains and $\tau_{\mathrm{BF}}$ compensates for the motor friction such that the closed loop dynamics takes the form

$$
\frac{1}{K_{\mathrm{P}}}\left(B \ddot{\theta}+K_{\mathrm{D}} \dot{\theta}+\tau(\theta, q)\right)=\theta_{d}-\theta .
$$

From (5) it can be seen that for $K_{\mathrm{P}} \rightarrow \infty, \theta \rightarrow \theta_{\mathrm{d}}$. We can conclude that for sufficiently high values of $K_{\mathrm{P}} \gg$ $\partial^{2} U(\theta, q) / \partial q^{2}$, the singular perturbation assumption in [18] is satisfied such that the motor position $\theta$ in (3) can be considered as control input. Note that due to the mechanical robustness requirement, compliant actuators are usually designed such that the value of the controller gain $K_{\mathrm{P}}$ can be chosen much higher than the instantaneous joint stiffness $\partial^{2} U(\theta, q) / \partial q^{2}$. As long as this assumption is fulfilled, it allows to treat the controller as well as the mechanical design for the link side dynamics completely separated from the choice of the servo units and the corresponding controller design.

\section{B. Switching based limit cycle control}

The basic concept of limit cycle generation for one joint which perfectly meets the requirements (ii) energy efficiency and (iii) robustness against plant parameters,switches the generalized motor position by a constant amount $\hat{\theta}$ triggered by a threshold $\epsilon_{\tau}$ on the generalized force $\tau$ [19], i. e.,

$$
\theta=\left\{\begin{array}{ll}
\operatorname{sign}(\tau) \hat{\theta} & \text { if }|\tau|>\epsilon_{\tau} \\
0 & \text { otherwise }
\end{array} .\right.
$$

Note that there exist a one-to-one correspondence between $\phi$ and $\tau$ such that the controller (6) can be equivalently formulated in terms of $\phi$. For all controller parameters satisfying $\hat{\theta}>2 \epsilon_{\phi}$, the controller asymptotically stabilizes a limit cycle [20]. Thereby, energy efficiency can be deduced from the property of the controller only performing positive

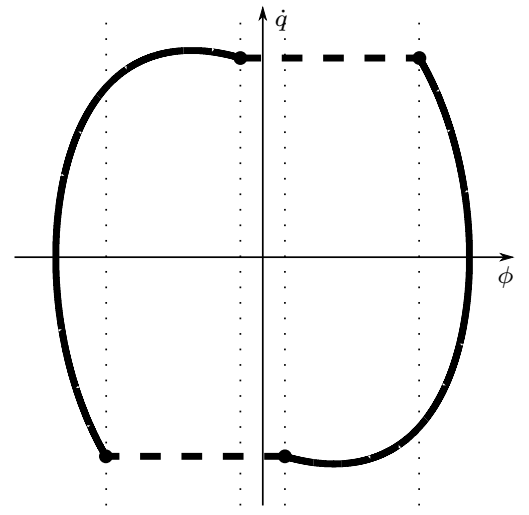

Fig. 1. Typical limit cycle resulting from the switching control (6). Each solid line represents the continuous motion of one half-cycle and each dashed line represents the switching.

work on the load. If either the spring deflection $\phi$ or the force $\tau$ can be measured, only structural informations (no plant parameters) are required to compute the control input $\theta$. An additional advantage of the control law is that no time derivatives of measured signals are required. Therefore, the control concept is very robust w.r.t. uncertainties of plant parameters.

\section{Discrete planning the controller parameters}

The basic problem addressed in this paper is to adapt the shape of the limit cycle to the locomotion task. A typical limit cycle resulting from the switching control (6) is depicted in Fig. 1. It should be taken into account that the requirements change from one (half-)cycle to the next. Due to the finite dynamics of the controller (6), also the control input $\theta$ has discrete states. The usually continuous problem of planning set points and controller parameters collapses to a discrete time planning problem. For the closed loop system (3) and (6) and for controller parameters $\hat{\theta}>2 \epsilon_{\phi}$, two switchings per cycle occur such that the trajectory in the state plane $(q, \dot{q})$ can be divided in two half-cycles. The problem of reaching a specific terminal state $(q, \dot{q})_{1}$ for a given initial state $(q, \dot{q})_{0}$ (both on the half-cycle) reduces to finding the controller parameters $\hat{\theta}$ and $\epsilon_{\phi}$. Thus, the planning problem becomes discrete in time.

\section{Multi-DEGREE-OF-FREEDOM HOPPING CONTROL}

In this section, we present a control methodology which extends the concept of limit cycle generation described in Sect. II-B to the case of legged floating base systems. Therefore, we first introduce the dynamic model. Then, we propose a coordinate transformation which reduces the dimensions of the control problem such that the limit cycle concept of Sect. II-B can be applied. Finally, we derive the finite dynamics which specifies the control input of the plant.

\section{A. Dynamic model}

The configuration of the legged floating base system is uniquely determined by $\boldsymbol{x}=\left(\begin{array}{ll}\boldsymbol{x}_{\mathrm{b}}^{T} & \boldsymbol{q}^{T}\end{array}\right)^{T}$ comprising the coordinates of the floating base $x_{\mathrm{b}} \in \mathbb{R}^{m}$ and the joint 

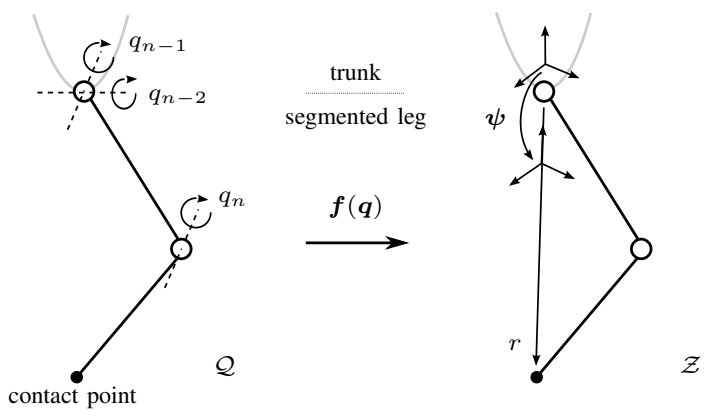

Fig. 2. Change of coordinates between joint coordinates $\boldsymbol{q} \in \mathcal{Q} \subset \mathbb{R}^{n \leq 3}$ and task coordinates $\boldsymbol{z}=\left(\boldsymbol{\psi}^{T}, r\right)^{T} \in \mathcal{Z} \subset \mathbb{R}^{n \leq 3}$.

coordinates of the legs $q \in \mathbb{R}^{n}$. In the following, we consider dynamic systems satisfying

$$
\begin{array}{r}
M(\boldsymbol{x}) \ddot{\boldsymbol{x}}+\boldsymbol{C}(\boldsymbol{x}, \dot{\boldsymbol{x}}) \dot{\boldsymbol{x}}+\boldsymbol{g}(\boldsymbol{x})= \\
\left(\begin{array}{c}
\mathbf{0} \\
\boldsymbol{\tau}(\boldsymbol{\theta}, \boldsymbol{q})-\boldsymbol{d}(\boldsymbol{q}, \dot{\boldsymbol{q}})
\end{array}\right)+\boldsymbol{\tau}_{\mathrm{ext}}
\end{array}
$$

Herein, $\boldsymbol{M}(\boldsymbol{x}) \in \mathbb{R}^{(m+n) \times(m+n)}$ represents the symmetric and positive definite inertia matrix, $\boldsymbol{C}(\boldsymbol{x}, \dot{\boldsymbol{x}}) \in$ $\mathbb{R}^{(m+n) \times(m+n)}$ represents the matrix of Coriolis/centrifugal terms, and $\boldsymbol{g}(\boldsymbol{x}) \in \mathbb{R}^{m+n}$ represents the vector of generalized gravitational forces. The generalized elastic forces $\boldsymbol{\tau}(\boldsymbol{\theta}, \boldsymbol{q}) \in$ $\mathbb{R}^{n}$ of the actuators are derived from a potential such that

$$
\boldsymbol{\tau}(\boldsymbol{\theta}, \boldsymbol{q})=-\frac{\partial U_{\tau}(\boldsymbol{\theta}, \boldsymbol{q})}{\partial \boldsymbol{q}},
$$

where $\boldsymbol{\theta} \in \mathbb{R}^{n}$ is considered as control input and $U_{\tau}(\boldsymbol{\theta}, \boldsymbol{q})$ is the total elastic potential energy. Furthermore, the generalized dissipative forces $\boldsymbol{d}(\boldsymbol{q}, \dot{\boldsymbol{q}}) \in \mathbb{R}^{n}$ are such that $\boldsymbol{d}(\boldsymbol{q}, \dot{\boldsymbol{q}})^{T} \dot{\boldsymbol{q}} \geq 0$ and $\boldsymbol{\tau}_{\text {ext }} \in \mathbb{R}^{m+n}$ summarizes the generalized external forces of the complete floating base system.

\section{B. Coordinate transformation}

Consider a single segmented leg of the legged floating base system, whose configuration can be determined by the joint coordinates $\boldsymbol{q} \in \mathcal{Q} \subset \mathbb{R}^{n \leq 3}$. Assume that the leg is such that directed distance between the rotational hip joint and the single contact point representing the foot can be described by a single coordinate $r \in \mathbb{R}$. The orientation of the vector connecting the center of rotation of the hip and the contact point represented in trunk coordinates can be parametrized by $\boldsymbol{\psi} \in \mathbb{R}^{n-1 \leq 2}$ (see, Fig. 2$)^{1}$. Then, the corresponding coordinate transformation can be expressed as

$$
z=\boldsymbol{f}(\boldsymbol{q})
$$

where $\boldsymbol{z}=\left(\boldsymbol{\psi}^{T}, r\right)^{T} \in \mathcal{Z} \subset \mathbb{R}^{n \leq 3}$. The control concept of Sect. II-B requires to observe a one-dimensional generalized force (or spring deflection) which reflects the elastic energy of the system. This force can be identified by considering the generalized elastic force $\tau$ in task coordinates of $\mathcal{Z}$. To obtain the corresponding transformation for the generalized

\footnotetext{
${ }^{1}$ The quantity $\psi$ is a scalar in the planar case and of dimension 2 in the 3 degree-of-freedom spatial leg.
}

force, let us differentiate the elastic potential $U_{\tau}$ w.r.t. $z$ which yields

$$
\boldsymbol{\tau}_{z}=-\left(\frac{\partial U_{\tau}(\theta, \boldsymbol{q})}{\partial \boldsymbol{q}} \frac{\partial \boldsymbol{q}}{\partial \boldsymbol{z}}\right)^{T}=\boldsymbol{J}_{z}(\boldsymbol{q})^{-T} \boldsymbol{\tau}(\boldsymbol{\theta}, \boldsymbol{q})
$$

Herein, $\boldsymbol{J}_{z}(\boldsymbol{q}) \in \mathbb{R}^{n \times n}$ is the Jacobian matrix corresponding to (9). In (10) the relation (8) has been used. The force which dominantly performs the work on the elasticities is the force component in the direction of $r$ (cf. spring loaded inverted pendulum (SLIP) model [21]). Therefore, we define the force component which triggers the switching of the controller:

$$
\tau_{z_{r}}=\left(\begin{array}{lll}
0 & \ldots & 1
\end{array}\right)_{1 \times n} \boldsymbol{J}_{z}(\boldsymbol{q})^{-T} \boldsymbol{\tau}(\boldsymbol{\theta}, \boldsymbol{q}) .
$$

Following the control concept of Sect. II-B, the control action will be a switching between generalized motor positions $\boldsymbol{\theta}_{z}^{\mathrm{TD}}$ and $\boldsymbol{\theta}_{z}^{\mathrm{TO}}$ both expressed w.r.t. the coordinate system of $\mathcal{Z}$. For the legged system, $\boldsymbol{\theta}_{z}^{\mathrm{TD}}$ is the control input of the touchdown and $\boldsymbol{\theta}_{z}^{\mathrm{TO}}$ is the control input of the takeoff. The control input

$$
\boldsymbol{\theta}_{z}^{\mathrm{TO}}=\boldsymbol{\theta}_{z, 0}^{\mathrm{TO}}+\left(\begin{array}{lll}
0 & \ldots & 1
\end{array}\right)_{1 \times n}^{T} \hat{r}
$$

comprises the "equilibrium" position $\boldsymbol{\theta}_{z, 0}^{\mathrm{TO}}$ and the switching amplitude $\hat{r}>0$ in the direction of $r$. From (12) it can be seen that the control input comprises two components: one for switching the elastic energy and one for switching the configuration of the leg. The parameter of the former component is $\hat{r}$ which is comparably with the switching amplitude $\hat{\theta}$ in the single joint case (cf. (6)). The later component switches between the takeoff configuration $\boldsymbol{\theta}_{z, 0}^{\mathrm{TO}}$ and the touchdown configuration $\boldsymbol{\theta}_{z, 0}^{\mathrm{TD}}=\boldsymbol{\theta}_{z}^{\mathrm{TD}}$. This component appears only in the multi-joint case.

In order to compute the "physical" control input $\boldsymbol{\theta}$, the inverse of the mapping (9) has to be applied, i. e.

$$
\boldsymbol{\theta}=\boldsymbol{f}^{-1}\left(\boldsymbol{\theta}_{z}\right)
$$

Remark 1: The inverse mapping (13) is defined for all $z \in \overline{\mathcal{Z}}:=\left\{\boldsymbol{z} \in \mathbb{R}^{n \leq 3} \mid z_{n}<r_{\max }\right\}$. In some cases it might be required that $\theta_{z_{n}}>r_{\max }$, i.e. a higher amount of energy has to be input into the system. Then, the inverse mapping can be approximated such that

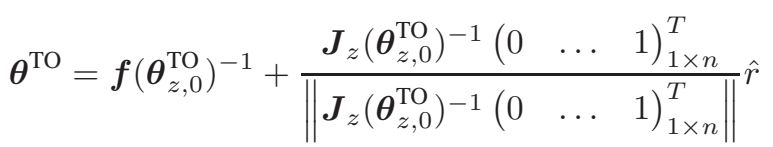

This is of course only possible if $\boldsymbol{\theta}_{z, 0}^{\mathrm{TO}} \in \overline{\mathcal{Z}}$.

\section{Finite controller dynamics}

On the basis of the coordinates derived in Sect. III-B, we introduce a switching controller which induces a jumping motion in a single compliantly actuated, segmented leg. The controller can be straightforwardly extended to multi-legged systems considering the concepts of virtual legs ${ }^{2}$ as proposed

\footnotetext{
${ }^{2}$ Virtual legs are groups of legs which are simultaneously in contact [1]
} 


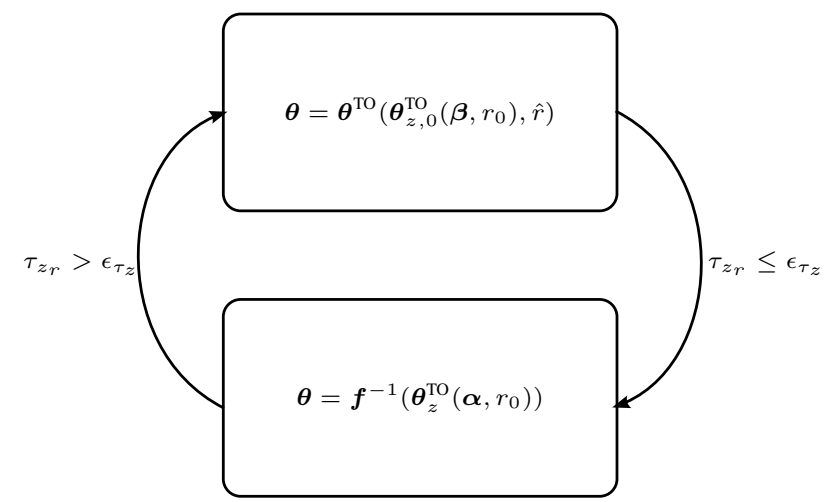

Fig. 3. Representation of the controller (15) as finite state machine. The controller switches only between two constant positions.

in [1]. Following the ideas of the controller (6) in Sect. II-B, the jumping controller takes the form

$\boldsymbol{\theta}\left(\boldsymbol{q}, \boldsymbol{\theta}_{-}\right)=\left\{\begin{array}{cc}\boldsymbol{\theta}^{\mathrm{TO}}\left(\boldsymbol{\theta}_{z, 0}^{\mathrm{TO}}\left(\boldsymbol{\beta}, r_{0}\right), \hat{r}\right) & \text { if } \tau_{z_{r}}\left(\boldsymbol{q}, \boldsymbol{\theta}_{-}\right)>\epsilon_{\tau_{z}} \\ \boldsymbol{f}^{-1}\left(\boldsymbol{\theta}_{z}^{\mathrm{TD}}\left(\boldsymbol{\alpha}, r_{0}\right)\right) & \text { if } \tau_{z_{r}}\left(\boldsymbol{q}, \boldsymbol{\theta}_{-}\right) \leq \epsilon_{\tau_{z}}\end{array}\right.$

Herein, we parametrize the takeoff and touchdown configuration by orientations of the leg axis $\boldsymbol{\beta} \in \mathbb{R}^{n-1 \leq 2}$ and $\alpha \in \mathbb{R}^{n-1 \leq 2}$, respectively, and a constant length of the leg axis $r_{0} \in \mathbb{R}_{\geq 0}$, i. e.,

$$
\begin{aligned}
\boldsymbol{\theta}_{z, 0}^{\mathrm{TO}} & =\left(\begin{array}{c}
\boldsymbol{\beta} \\
r_{0}
\end{array}\right) \\
\boldsymbol{\theta}_{z}^{\mathrm{TD}} & =\left(\begin{array}{c}
\boldsymbol{\alpha} \\
r_{0}
\end{array}\right) .
\end{aligned}
$$

Note that $\boldsymbol{\theta}^{\mathrm{TO}}$ in (15) can be computed either by (12) or (14), while the latter is an approximation (Taylor approximation of first order) of the coordinate transformation (13). The controller (15) switches the motor position $\boldsymbol{\theta}$ if the generalized force $\tau_{z_{r}}\left(\boldsymbol{q}, \boldsymbol{\theta}_{-}\right)$defined by (11) overshoots or undershoots the threshold $\epsilon_{\tau_{z}} \in \mathbb{R}_{\geq 0}$. Note that the generalized force $\tau_{z_{r}}\left(\boldsymbol{q}, \boldsymbol{\theta}_{-}\right)$depends on the motor position $\boldsymbol{\theta}$ which is also the output of the switching controller (15). Therefore, $\boldsymbol{\theta}_{-}$ represents the state of the controller before the switching. Comparing the switching laws (6) and (15) it can be seen that the jumping controller (15) contains the one sided ${ }^{3}$ version of the single joint controller (6). Additionally, the jumping controller accounts for the multiple degrees-of-freedom by switching the configuration of the leg.

The simplicity of the controller structure can be seen from the representation as finite state machine as depicted in Fig. 3. The state machine comprises two states, each corresponding to a constant (desired) motor position. The controller parameters of one jumping cycle are listed in Table I.

\section{Velocity control}

The jumping controller (15) can be used to implement a simple, but very effective velocity control similar to the

\footnotetext{
${ }^{3}$ The jumping controller has only the two states $r_{0}$ and $r_{0}+\hat{r}$ while the single joint controller has the three states $-\hat{\theta}, 0,+\hat{\theta}$.
}

TABLE I

CONTROLLER PARAMETERS OF ONE JUMPING CYCLE.

\begin{tabular}{l|l} 
takeoff orientation & $\boldsymbol{\beta}$ in rad \\
\hline touchdown orientation & $\boldsymbol{\alpha}$ in rad \\
\hline initial length of leg axis & $r_{0}$ in $\mathrm{m}$ \\
\hline switching amplitude & $\hat{r}$ in $\mathrm{m}$ \\
\hline switching threshold & $\epsilon_{\tau_{z}}$ in $\mathrm{N}$
\end{tabular}

principle of [1]. The idea of the velocity regulator is updating the parameters of the jumping controller (15) once per jumping cycle to iteratively converge to the desired forward jumping velocity. Therefore, we consider $\boldsymbol{\alpha}$ in (15) as input and chose $\boldsymbol{\beta}=-\boldsymbol{\alpha}$. The remaining controller parameters $\epsilon_{\tau_{z}}, r_{0}$, and $\hat{r}$ are kept constant. Then, the iterative update law can be expressed as

$$
\boldsymbol{\alpha}(k)=\boldsymbol{\alpha}(k-1)+\boldsymbol{K}_{\boldsymbol{v}}\left(\overline{\boldsymbol{v}}(k)-\boldsymbol{v}_{\mathrm{d}}\right),
$$

where $\boldsymbol{K}_{\boldsymbol{v}} \in \mathbb{R}^{(n-1 \leq 2) \times(n-1 \leq 2)}$ is a symmetric and positive definite gain matrix and the average velocity

$$
\overline{\boldsymbol{v}}(k)=\frac{\boldsymbol{x}_{\mathrm{b}}(k)-\boldsymbol{x}_{\mathrm{b}}(k-1)}{t(k)-t(k-1)}
$$

represents the feedback of the jumping cycle $k$.

\section{Discrete PLANNing}

The input to the controller (15) introduced in Sect. III is only a set of parameters, i.e., it is not a trajectory of reference values as common for many approaches based on tracking control [22]. This set of controller parameters (see, Table I) defines the motion of a complete jumping cycle, for instance, it defines the motion from one touchdown to the next touchdown. As a result, the problem of planning a trajectory of reference values collapses to the problem of planning a set of controller parameters for each jumping step. Since the planning problem is discrete in time, we call it discrete planning.

\section{A. Problem formulation}

The considered problem is finding the controller parameters which bring the system from a given initial state to a desired final state. Thereby, it is assumed that the states are connected via the jumping motion generated by the controller of Sect. III. As an example, we consider the parametrization of the jumping cycle as depicted in Fig. 4. Herein, the jumping cycle starts with the touchdown event $\mathrm{TD}^{i-1}$ after the preceding flight phase and ends with the touchdown event $\mathrm{TD}^{i}$ of the current cycle. At the initial event of the cycle $\mathrm{TD}^{i-1}$ it is assumed that the leg configuration is in a steady state, i. e., $\dot{\boldsymbol{q}}=\mathbf{0}$, such that the motor position $\boldsymbol{\theta}\left(\boldsymbol{\alpha}^{i-1}, r_{0}^{i-1}\right)$ and link position $\boldsymbol{q}\left(\boldsymbol{\alpha}^{i-1}, r_{0}^{i-1}\right)$ are completely determined by $\boldsymbol{\alpha}^{i-1}$ and $r_{0}^{i-1}$. Thus, $\boldsymbol{\alpha}^{i-1}, r_{0}^{i-1}$ and the velocity of the complete system $\boldsymbol{v}^{\mathrm{TD}, i-1}$ determine the complete state of the jumping system at $\mathrm{TD}^{i-1}$. Now, given this initial state and assuming that the point in contact is not slipping during the stance phase, the controller parameters $\boldsymbol{\alpha}^{i}, \boldsymbol{\beta}^{i}$, $r_{0}^{i}, \hat{r}^{i}$, and $\epsilon_{\tau_{z}}^{i}$ determine the difference in the foot position $\Delta \boldsymbol{p}^{i}$ which result during the flight phase. The final state at 


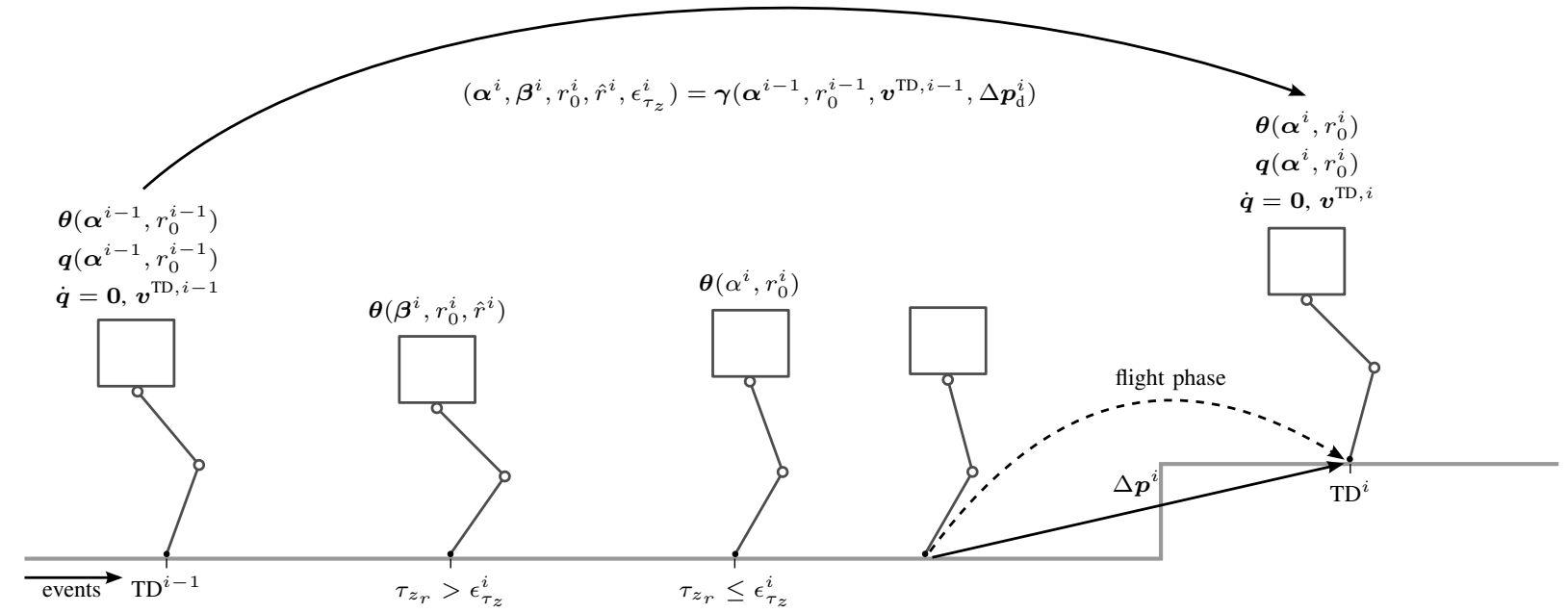

Fig. 4. Parametrization of one jumping cycle from touchdown of cycle $i-1$ to touchdown of cycle $i$.

$\mathrm{TD}^{i}$ is determined by the controller parameters $\boldsymbol{\alpha}^{i}$ and $r_{0}^{i}$ together with the velocity at touchdown $\boldsymbol{v}^{\mathrm{TD}, i}$ which in turn represent the initial conditions for the next jumping cycle. In summary, the problem can be formulated as follows: Given, the controller parameters of the last cycle $\boldsymbol{\alpha}^{i-1}$ and $r_{0}^{i-1}$, and the velocity at touchdown $\boldsymbol{v}^{\mathrm{TD}, i-1}$ which together determine the initial state of the system, the problem is finding a mapping $\boldsymbol{\gamma}$ which returns the controller parameters $\boldsymbol{\alpha}^{i}, \boldsymbol{\beta}^{i}$, $r_{0}^{i}, \hat{r}^{i}$, and $\epsilon_{\tau_{z}}^{i}$, corresponding to a desired jump of $\boldsymbol{p}_{\mathrm{d}}^{i}$ in the foot position. Formally, this mapping takes the form

$$
\left(\boldsymbol{\alpha}^{i}, \boldsymbol{\beta}^{i}, r_{0}^{i}, \hat{r}^{i}, \epsilon_{\tau_{z}}^{i}\right)=\gamma\left(\boldsymbol{\alpha}^{i-1}, r_{0}^{i-1}, \boldsymbol{v}^{\mathrm{TD}, i-1}, \Delta \boldsymbol{p}_{\mathrm{d}}^{i}\right) \text {. }
$$

\section{B. Generating the mapping}

Due to the hybrid and nonlinear nature of the system and also due to limits in the actuators (e.g., motor torque and velocity limits), the mapping (20) is highly nonlinear and discontinuous. Therefore, numerical search methods are required to compute the map for a required grid of input variables. A method which fits to this type of problems is the Covariance Matrix Adaption (CMA) algorithm which is an evolutionary algorithm for non-linear and non-convex blackbox optimization problems [23].

Our control model introduces the free parameters $\boldsymbol{\alpha}^{i}, \boldsymbol{\beta}^{i}, r_{0}^{i}, \hat{r}^{i}$ and $\epsilon_{\tau_{z}}^{i}$ to control the movement of the leg. For simplication of the mapping we exclude $r_{0}^{i}$ and $\epsilon_{\tau_{z}}^{i}$ from the optimization and configure them manually with constant values. This is primarily possible because they are mostly independent of the other parameters and are very stable for a large range of values. The remaining three parameters are optimized using CMA with step size $\sigma=1$ and population size $\lambda=5$ over 30 generations.

The target of the optimization is a cost functions tailored to a desired jumping behavior. We distinguish between two types of jumps, namely a jump on the spot and a forward jump. The basic cost function of the jumping cycle $i$ is chosen as

$$
J\left(\boldsymbol{\alpha}^{i}, \boldsymbol{\beta}^{i}, \hat{r}^{i}\right)=\sum_{j=1}^{n} \omega_{j}\left|\Delta p_{\mathrm{d}, j}^{i}-\Delta p_{j}^{i}\right|
$$

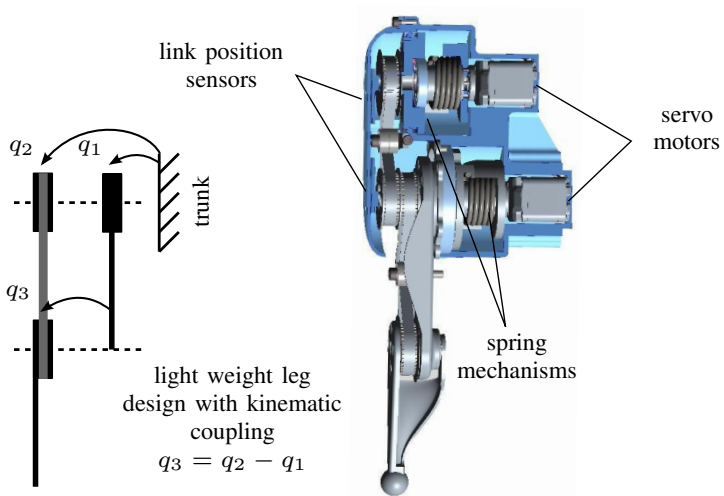

Fig. 5. System design of the compliantly actuated leg considered in the experiments.

Herein, $\Delta p_{\mathrm{d}, j}^{i}$ are the desired jump distances and $\Delta p_{j}$ the actual jump distances, for $j=1, \ldots, n$, according to the notation introduced in Sect. IV-A. In our particular planar case considered in the experiments, $\omega_{1}$ and $\omega_{2}$ are weighting parameters which we set to 0.6 and 0.4 , respectively, to prioritize a small deviation in the horizontal direction over a small deviation in vertical direction. For both jumping types, $\Delta p_{\mathrm{d}, 2}$ is set to the estimated maximum jumping height of the leg while $\Delta p_{\mathrm{d}, 1}=0$ is set to zero for the case of a jump on the spot and to a desired distance $\Delta p_{\mathrm{d}, 1} \neq 0$ for a forward jump.

\section{EXPERIMENTS}

\section{A. System design}

The design of the compliantly actuated leg follows the concept of low-cost and efficiency. Therefore, the main parts of the mechatronic system are the spring mechanisms which implement a constant stiffness $K \approx 1.5 \mathrm{Nm} / \mathrm{rad}$ and are serially connected to position controlled servo units (HerkuleX DRS-201). The output of one series elastic actuator (SEA) is directly connected to the thigh link and the output of the other SEA is connected via belt drives to the knee joint. This results in a kinematic coupling of the of the degrees- 


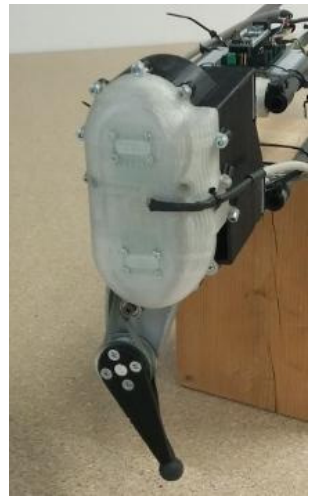

Fig. 6. Compliantly actuated leg prototype.

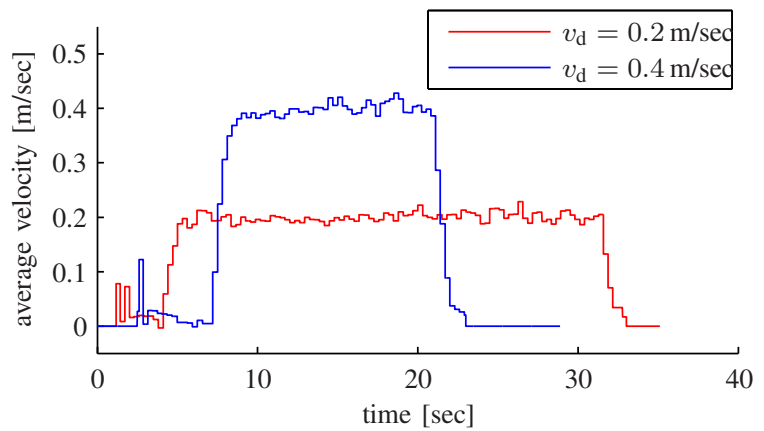

Fig. 7. Velocity control experiments. Horizontal velocity averaged over each jumping cycle is depicted for two different reference values of the velocity controller. At the beginning and at the end of the experiment the reference value is always zero.

of-freedom of the hip and knee joint as depicted in Fig. 5 which reduces the weight of the leg. The servo units as well as the output shafts of the SEAs are equipped with position sensors such that the elastic torques can be estimated via the SEA stiffness. All parts except the parts of the power train are produced by 3D printing. Figure 6 shows a picture of the prototype. For the experiments, the trunk is mounted to a boom which constrains the motion to a horizontal and a vertical position on the orbit, while the rotation is locked.

\section{B. Results}

The concepts of targeted jumping have been experimentally tested on the compliantly actuated leg prototype described in Sect. V-A. The first tests evaluate the performance of the velocity control described in Sect. III-D. The second tests validate the concept of discrete planning introduced in Sect. IV.

As can be seen from Fig. 7, the controller is able to stabilize jumping motions for several desired velocities including zero. This indicates that the presented approach may be seen as a generalization of the pogo stick introduced in [1] to the segmented leg.

In order to plan a desired jumping sequence, the cost function (21) has been evaluated for given initial system states and some parameter sets. In our first attempt we have chosen to evaluate the cost function using a dynamics simulation of the leg to be ably to quickly evaluate a large quantity of

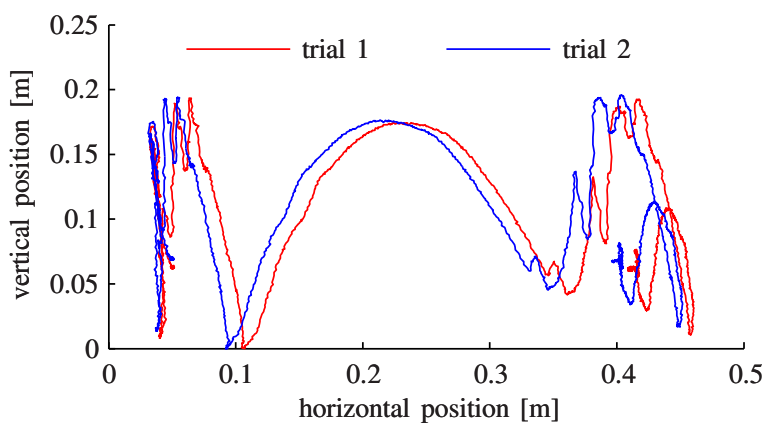

Fig. 8. Sequence of four directed jumps. Motion of two trials in horizontal and vertical direction on the orbit of the boom is shown.

parameter sets and then test the optimized parameters on the hardware system. We optimized sequences of four jumps in a row, whereby the end state of a jump was used as the initial state of the succeeding jump. The resulting parameter set has been tested on the hardware system. Fig. 8 depicts paths of the trunk motion in the sagittal plane (horizontal versus vertical position of the trunk). As can be seen from Fig. 8, a desired jumping pattern can be generated on the real system with a good repeatability, despite the usual limitations of low-cost hardware and only unprecise knowledge of plant parameters.

The velocity controller considers feedback of the plant and therefore reaches the control goal accurately. In contrast, the discrete planer as it is implemented in the experiments generates the jumping sequence in an open-loop manner and therefore performs less accurate on the real plant. In order to improve the accuracy of the discrete planer, the cost functional (21) should be evaluated on the real plant incorporating direct feedback of the plant. This requires repeatability and the ability of long term usage of the hardware system. While the former has already been shown to be valid for our compliantly actuated leg prototype, the latter will be part of our future engineering work.

The attached video shows the performed experiments.

\section{CONCLUSION}

The main contribution of the paper is exploring the opportunities offered by the concept of exciting the resonance mode of an elastic body by a bang-bang controller. Only one switching per cycle is used to control the energy of the system and to switch between landing and take-off configurations. The experiments demonstrate that the bangbang controller performs reliably on a new rapid prototyped leg, for which only rough dynamic models were available. Velocity control can be achieved by simply controlling the angle of attack of the leg. Moreover, we introduced a discrete optimization approach for generating sequences of steps of different length and height. Only three to five parameters need to be configured once per step, leading to fast optimization procedures. This is mainly due to the simplicity and discrete nature of the controller, while its robustness to uncertain parameters ensures stability of the steps under real conditions. Addressing the balancing for the leg prototype, 
extension to online planning and to $3 \mathrm{D}$, quadruped robots are the next steps.

\section{REFERENCES}

[1] M. H. Raibert, Legged Robots That Balance. The MIT Press, 1986.

[2] J. Pratt, "Exploiting inherent robustness and natural dynamics in the control of bipedal walking robots," Ph.D. dissertation, Massachusetts Institute of Technology, 2000.

[3] A. Seyfarth, H. Geyer, M. Gnther, and R. Blickhan, "A movement criterion for running." Journal of Biomechanics, vol. 35, no. 5, pp. 649-55, 2002.

[4] H. Geyer, A. Seyfarth, and R. Blickhan, "Compliant leg behavior explains basic dynamics of walking and running," Proceedings of the Royal Society B, vol. 273, pp. 2861-2867, Nov. 2006.

[5] J. Rummel, Y. Blum, H. M. Maus, C. Rode, and A. Seyfarth, "Stable and robust walking with compliant legs," in Proc. of IEEE Int. Conf. on Robotics and Automation, 2010, pp. 5250-5255.

[6] J. W. Hurst, The role and implementation of compliance in legged locomotion. ProQuest, 2008.

[7] K. Sreenath, H.-W. Park, and J. W. Grizzle, "Embedding active force control within the compliant hybrid zero dynamics to achieve stable, fast running on MABEL," The International Journal of Robotics Research, 2013.

[8] G. Folkertsma, A. van der Schaft, and S. Stramigioli, "Powercontinuous synchronisation of oscillators: A novel, energy-free way to synchronise dynamical systems," in IEEE Int. Conf. on Robotics and Automation, May 2014, pp. 1493-1498.

[9] H. Geyer and H. Herr, "A muscle-reflex model that encodes principles of legged mechanics produces human walking dynamics and muscle activities," Neural Systems and Rehabilitation Engineering, IEEE Transactions on, vol. 18, no. 3, pp. 263-273, 2010.

[10] H.-M. Maus, J. Rummel, and A. Seyfarth, "Stable upright walking and running using a simple pendulum based control scheme," in Advances in Mobile Robotics: Proc. 11th Int. Conf. Climbing and Walking Robots. Coimbra, Portugal: World Scientific, 2008, pp. 623-629.

[11] D. J. Braun, M. Howard, and S. Vijayakumar, "Exploiting variable stiffness in explosive movement tasks," in Robotics: Science and Systems, 2011.
[12] D. Braun, F. Petit, F. Huber, S. Haddadin, P. van der Smagt, A. AlbuSchaffer, and S. Vijayakumar, "Optimal torque and stiffness control in compliantly actuated robots," in Proc. IEEE/RSJ Int. Conf. on Intelligent Robots and Systems. IEEE, 2012.

[13] S. Haddadin, F. Huber, and A. Albu-Schäffer, "Optimal control for exploiting the natural dynamics of variable stiffness robots," in IEEE Int. Conf. on Robotics and Automation, 2012.

[14] D. Braun, F. Petit, F. Huber, S. Haddadin, P. van der Smagt, A. AlbuSchaffer, and S. Vijayakumar, "Robots driven by compliant actuators: Optimal control under actuation constraints," Robotics, IEEE Transactions on, vol. 29, no. 5, pp. 1085-1101, Oct 2013.

[15] D. Lakatos, M. Görner, F. Petit, A. Dietrich, and A. Albu-Schäffer, "A modally adaptive control for multi-contact cyclic motions in compliantly actuated robotic systems," in Proc. IEEE/RSJ Int. Conf. on Intelligent Robots and Systems, 2013, pp. 5388-5395.

[16] D. Lakatos, G. Garofalo, A. Dietrich, and A. Albu-Schäffer, "Jumping control for compliantly actuated multilegged robots," in IEEE Int. Conf. on Robotics and Automation, 2014, pp. 4562-4568.

[17] D. Lakatos and A. Albu-Schaffer, "Neuron model interpretation of a cyclic motion control concept," in Biomedical Robotics and Biomechatronics (2014 5th IEEE RAS \& EMBS International Conference on. IEEE, 2014, pp. 905-910.

[18] P. Kokotovic, H. Khalil, and J. O'Reilly, Singular Perturbation Methods in Control: Analysis and Design. Academic Press, London, 1986.

[19] D. Lakatos, F. Petit, and A. Albu-Schäffer, "Nonlinear oscillations for cyclic movements in human and robotic arms," IEEE Transactions on Robotics, vol. 30, no. 4, pp. 865-879, 2014.

[20] D. Lakatos and A. Albu-Schäffer, "Switching based limit cycle control for compliantly actuated second-order systems," in Proceedings of the 19th IFAC World Congress, 2014, pp. 6392-6399.

[21] R. Blickhan, "The spring-mass model for running and hopping," Journal of Biomechanics, vol. 22, pp. 1217-1227, 1989.

[22] C. Gehring, S. Coros, M. Hutter, M. Bloesch, M. Hoepflinger, and R. Siegwart, "Control of dynamic gaits for a quadrupedal robot," in IEEE Int. Conf. on Robotics and Automation, May 2013, pp. 32873292.

[23] N. Hansen, "The CMA evolution strategy: a comparing review," in Towards a new evolutionary computation. Advances on estimation of distribution algorithms, J. Lozano, P. Larranaga, I. Inza, and E. Bengoetxea, Eds. Springer, 2006, pp. 75-102. 